\title{
Applications of the CRISPR-Cas9 System in Generation of Model Cell Lines for Cancer Biology
}

\section{Hossein Arabameri ${ }^{1}$, Saghar Pahlavanneshan ${ }^{2,3}$, Mahmood} Tavallaei ${ }^{1,{ }^{*}}$, Mohsen Basiri ${ }^{2, *}$

${ }^{1}$ Genetic Research Center, Baqiyatallah University of Medical Sciences, Tehran, Iran

${ }^{2}$ Department of Stem Cells and Developmental Biology, Cell Science Research Center, Royan Institute for Stem Cell Biology and Technology, ACECR, Tehran, Iran

${ }^{3}$ Department of Molecular Genetics, Faculty of Biological Sciences, Tarbiat Modares University, Tehran, Iran

* Corresponding authors: Mahmood Tavallaei, Applications of the CRISPRCas9 System in Generation of Model Cell Lines for Cancer Biology. E-mail:

DOI: $10.21859 /$ mci-supp-38 mahmoodtavallaei@yahoo.com,basiri@royaninstitute.org

\section{Keywords:}

Cancer

Cell Line

CRISPR CAS9

\begin{abstract}
Introduction Cancer is known to be the second leading cause of death in the world after the cardiovascular disease. Therefore, it is important to study the various factors involved in cancer. In this regard, animal and cell models can be used to examine the different stages of cancer, as well as its prevention and treatment. In the present study, we tried to stablish a genome editing pipeline to generate genome edited cancer cell lines using CRISPR CAS9 technology.

Materials and Methods: We seeded about 40,000 cells in a 4-well plate and transfected CRISPR CAS9 constructs by PEI to A549 cells on the next day followed by a brief selection with puromycin. We allowed the remaining cells to multiply and form colonies then we separated and transferred them in new plates using ring cloning method. On the obtained colonies, we examined the function of the CRISPR, precisely designed the primer for the CRISPR target area and sequenced the PCR product.

Results: In our study, we were able to colonize CRISPR-modified single cells and propagate them into permanent cell lines harboring the modified CRISPR target. Using transient expression of the antibiotic resistance gene, we successfully enriched the colonies with genome modified clones to obtain a higher efficiency.

Conclusions: Proposed method provides a convenient means to generate different genome-edited cancer cell lines which can be used to study the function of any candidate gene in various stages of cancer, including metastasis, which plays a major role in increasing mortality in cancer patients.
\end{abstract}

